\title{
AVERAGING OF MAGNETIC FIELDS AND APPLICATIONS
}

\author{
AYMAN KACHMAR AND MOHAMMAD WEHBE
}

\begin{abstract}
We estimate the magnetic Laplacian energy norm in appropriate planar domains under a weak regularity hypothesis on the magnetic field. Our main contribution is an averaging estimate, valid in small cells, allowing us to pass from non-uniform to uniform magnetic fields. As a matter of application, we derive new upper and lower bounds of the lowest eigenvalue of the Dirichlet Laplacian which match in the regime of large magnetic field intensity. Furthermore, our averaging technique allows us to estimate the non-linear Ginzburg-Landau energy, and as a byproduct, yields a non-Gaussian trial state for the Dirichlet magnetic Laplacian.
\end{abstract}

\section{CONTEnTs}

1. Introduction

1.1. The magnetic field

1.2. The averaging estimate

1.3. The Dirichlet magnetic Laplacian

1.4. The Ginzburg-Landau functional

1.5. Organization of the paper

2. Preliminaries and notation

Asymptotic order

The averaged magnetic field

3. Averaging of the magnetic field

4. Approximation of the quadratic form

5. Magnetic Laplacian

5.1. Upper bound

5.2. Lower bound

5.3. Proof of Theorem 1.2

6. The Ginzburg-Landau model

6.1. Lower bound of GL energy

6.2. Upper bound of GL energy

6.3. Proof of Theorem 1.3

6.4. Further remarks

6.5. Application: The Dirichlet Laplacian

Acknowledgments

References

\section{INTRODUCTION}

The spectral properties of magnetic Schrödinger operators with minimal regularity assumptions on the magnetic field, magnetic potential and electrical potential, have been central since decades [14. Averaging of magnetic fields was also a valuable tool to study such spectral properties, notably the question of existence of a compact resolvent [13].

Date: November 30, 2021.

Mathematics Subject Classification (2010): 35B40, 35P15, 35Q56. 
In this paper, we study the averaging of magnetic fields in the context of spectral asymptotics (large field/semi-classical asymptotics). Our estimates will allow us to capture the leading order term in the large field asymptotics for the ground state energy of the magnetic Laplacian with a Dirichlet condition, via the essential infimum of the scalar magnetic field, under a weak regularity hypothesis.

1.1. The magnetic field. Consider a real-valued function

$$
B \in H^{1}\left(\mathbb{R}^{2}\right) .
$$

The function $B$ stands for a magnetic field (more precisely this is the vertical magnetic field with non-uniform intensity $B$, i.e. $B \vec{z}$ ). We introduce the corresponding magnetic potential $\mathbf{A}$ as follows

$$
\mathbf{A}(x)=\left(A_{1}(x), A_{2}(x)\right):=2 \int_{0}^{1} B(s x) \mathbf{A}_{0}(s x) d s \quad\left(x \in \mathbb{R}^{2}\right),
$$

where $\mathbf{A}_{0}$ is the canonical magnetic potential, satisfying curl $\mathbf{A}_{0}=1$ and defined as follows

$$
\mathbf{A}_{0}(x)=\frac{1}{2}\left(-x_{2}, x_{1}\right) \quad\left(x=\left(x_{1}, x_{2}\right) \in \mathbb{R}^{2}\right) .
$$

Clearly, $\mathbf{A} \in H^{2}\left(\mathbb{R}^{2}\right)$ and

$$
\operatorname{curl} \mathbf{A}:=\partial_{x_{1}} A_{2}-\partial_{x_{2}} A_{1}=B \text { in } \mathbb{R}^{2} .
$$

There are many other reasonable choices for the magnetic potential generating the magnetic field $B$, e.g. $\mathbf{A}+\nabla \chi$ for any smooth function $\chi$.

The aim of this paper is to estimate quantities of the form

$$
\int_{U}|(\nabla-i \sigma \mathbf{A}) u|^{2} d x
$$

where $\sigma \in \mathbb{R}, U$ is an appropriate convex subset of $\mathbb{R}^{2}$, typically a square or a disc of small diameter compared to the parameter $\sigma$, and $u \in H^{1}(U)$. Such questions naturally occur in many problems of mathematical physics, such as superconductivity [6], liquid crystals [8] and the theory of Schrödinger operators [18. The case of a smooth $\mathbf{A}$ is well developed in the literature, so our aim here is to address this question for the less regular case where $B \in H^{1}\left(\mathbb{R}^{2}\right)$ (i.e. $\mathbf{A} \in H^{2}\left(\mathbb{R}^{2}\right)$ ). This is related to [6, Sec. 16.6.1, Open Problem 9] and [16, Problem 2.2.9].

Our approach to approximate the quantity in (1.5) is through an averaging technique which will allow us to pass from $\mathbf{A}$ generating the non-smooth field $B$, to $\mathbf{A}_{\mathrm{av}}$ generating a constant field $B_{\mathrm{av}}$. The approximation will be valid in the regime of large field intensity, $\sigma \rightarrow+\infty$, and small domain $U, \operatorname{diam}(U) \rightarrow 0(\operatorname{diam}(U)$ stands for the diameter of $U)$. The precise statement will be given in Theorem 1.1 and Proposition 4.1 below.

\subsection{The averaging estimate. Assume that}

$$
x_{0} \in U \subset \mathbb{R}^{2} \text { and } U \text { is open, convex and bounded. }
$$

We denote by $|U|$ the area of $U$, and by $\operatorname{diam}(U)$, the diameter of $U$. We introduce the new magnetic potential

$$
\mathbf{A}_{\text {new }}^{U}(x)=2 \int_{0}^{1} B\left(s\left(x-x_{0}\right)+x_{0}\right) \mathbf{A}_{0}\left(s\left(x-x_{0}\right)\right) d s,
$$

where $\mathbf{A}_{0}$ is the canonical magnetic potential introduced in (1.3).

Note that, on $U$, curl $\mathbf{A}_{\text {new }}^{U}=B=\operatorname{curl} \mathbf{A}$, where $\mathbf{A}$ is the magnetic potential in (1.2). So there exists a function $\varphi^{U} \in H^{1}(U)$ such that

$$
\mathbf{A}=\mathbf{A}_{\text {new }}^{U}-\nabla \varphi^{U} \quad \text { on } U .
$$

We introduce the average of the magnetic field $B$ in $U$ as follows

$$
B_{\mathrm{av}}^{U}=\frac{1}{|U|} \int_{U} B(x) d x .
$$


It is then natural to introduce the average magnetic potential

$$
\mathbf{A}_{\mathrm{av}}^{U}(x)=B_{\mathrm{av}}^{U} \mathbf{A}_{0}\left(x-x_{0}\right)=2 B_{\mathrm{av}}^{U} \int_{0}^{1} \mathbf{A}_{0}\left(s\left(x-x_{0}\right)\right) d s,
$$

which generates the constant averaged magnetic field, curl $\mathbf{A}_{\mathrm{av}}^{U}=B_{\mathrm{av}}^{U}$. Theorem 1.1 below establishes that the magnetic potential $\mathbf{A}_{\mathrm{av}}^{U}$ is a good approximation of $\mathbf{A}_{\text {new }}$ in the convex domain $U$.

Theorem 1.1. Let $B \in H^{1}\left(\mathbb{R}^{2}\right)$. For every domain $U \subset \mathbb{R}^{2}$ satisfying (1.6), the following inequality holds,

$$
\int_{U}\left|\mathbf{A}_{\mathrm{new}}^{U}(x)-\mathbf{A}_{\mathrm{av}}^{U}(x)\right|^{2} d x \leq 8 \delta^{4}\|\nabla B\|_{L^{2}(U)}^{2}
$$

where $\delta=\operatorname{diam}(U), \mathbf{A}_{\text {new }}^{U}$ and $\mathbf{A}_{\mathrm{av}}^{U}$ are introduced in (1.7) and (1.10) respectively.

1.3. The Dirichlet magnetic Laplacian. As a consequence of Theorem 1.1, we can estimate the lowest eigenvalue, $\lambda(\sigma, \mathbf{A} ; \Omega)$, of the Dirichlet magnetic Laplacian $-(\nabla-i \sigma \mathbf{A})^{2}$ in $L^{2}(\Omega)$, for a domain $\Omega$ with a smooth $C^{1}$ boundary. Studying the strong field asymptotics, the essential infimum of the function $B$ in $\Omega$ shows up; this is the quantity introduced as follows

$$
m_{0}(B ; \Omega):=\underset{x \in \Omega}{\operatorname{essinf}} B(x)=\sup \{c \in \mathbb{R}: B(x) \geq c \text { a.e. on } \Omega\} .
$$

The variational min-max principle allows us to express the eigenvalue as follows 1 (when $\Omega$ is bounded)

$$
\lambda(\sigma, \mathbf{A} ; \Omega)=\inf _{u \in H_{0}^{1}(\Omega) \backslash\{0\}} \frac{\|(\nabla-i \sigma \mathbf{A}) u\|_{L^{2}(\Omega)}^{2}}{\|u\|_{L^{2}(\Omega)}^{2}} .
$$

Now we state our new estimates on the eigenvalue $\lambda(\sigma, \mathbf{A} ; \Omega)$.

Theorem 1.2. Assume that $\Omega=\bigcup_{i=1}^{N} \Omega_{i}$ where $N \geq 1$ is a positive integer, the sets $\overline{\Omega_{i}}$ are pairwise disjoint, and each $\Omega_{i}$ is a bounded connected domain of $\mathbb{R}^{2}$ such that $\partial \Omega_{i}$ consists of a finite number of smooth $C^{1}$ closed curves.

If $B \in H^{1}(\Omega)$ and the essential infimum in (1.11) is positive, then the lowest eigenvalue in (1.12) satisfies

$$
m_{0}(B ; \Omega) \sigma \leq \lambda(\sigma, \mathbf{A} ; \Omega) \leq m_{0}(B ; \Omega) \sigma+o(\sigma) \quad(\sigma \rightarrow+\infty) .
$$

The content of Theorem 1.2 is consistent with the known estimates for a smooth magnetic field (see [1]), in which case the essential infimum becomes

$$
m_{0}(B ; \Omega)=\min _{x \in \bar{\Omega}} B(x),
$$

and the remainder term $o(\sigma)$ can be explicitly controlled.

The non-asymptotic lower bound, $\lambda(\sigma, \mathbf{A} ; \Omega) \geq \sigma m_{0}(B ; \Omega)$, follows by a standard argument. The matching upper bound, $\lambda(\sigma, \mathbf{A} ; \Omega) \leq m_{0}(B ; \Omega) \sigma+o(\sigma)$, follows by constructing a trial state; the produced errors are controlled by the averaging estimate of Theorem 1.1 .

The novelty in Theorem 1.2 is establishing its validity in the weakly regular situation when (1.1) holds. This prevents us of deducing it from other works treating non-uniform magnetic fields, like smooth magnetic fields [2, 11, 15, 18, $B \in C^{0, \alpha}\left(\mathbb{R}^{2}\right)$, or step magnetic fields [3, 12].

It would be desirable to establish Theorem 1.2 under the much weaker hypothesis, $B \in L^{2}(\Omega)$. This is motivated by the current Theorem 1.2 and the existing results when $B$ is a step function [3, 12]. However, knowing $B \in L^{2}(\Omega)$ without further regularity, our averaging estimate

\footnotetext{
${ }^{1}$ The definition of the eigenvalue $\lambda(\sigma, \mathbf{A} ; \Omega)$ requires a vector field $\mathbf{A}$ (and consequently a magnetic field $B$ ) defined on $\Omega$, not the whole space $\mathbb{R}^{2}$. Our assumption on the domain $\Omega$ allows us to extend functions in the Sobolev space $H^{1}(\Omega)$ to functions in the space $H^{1}\left(\mathbb{R}^{2}\right)$, so that starting with $B \in H^{1}\left(\mathbb{R}^{2}\right)$ is not really a restriction. Our proofs require to deal with the value of the magnetic field outside the set $\Omega$.
} 
in Theorem 1.1 will be out of reach, thereby preventing us from proving the upper bound in Theorem 1.2 without the additional property $\nabla B \in L^{2}\left(\Omega ; \mathbb{R}^{2}\right)$.

1.4. The Ginzburg-Landau functional. Our averaging mechanism is robust in the study of the non-linear Ginzburg-Landau functional (see Theorem 1.3 below), which also contributes to the proof of Theorem 1.2 (by providing us with a useful trial state). Under the regularity assumption (1.1) on $B$, our contribution adds to the mainstream of understanding the role of non-uniform magnetic fields in the Ginzburg-Landau model [2, 5, 9, 10, 17]. Handling the particularities of our regularity hypothesis in (1.1) would not be possible without the averaging estimate of Theorem 1.1.

We restrict our study to a bounded domain $\Omega \subset \mathbb{R}^{2}$ which we assume connected and with a smooth boundary consisting of a finite number of smooth curves of class $C^{1}$. More precisely, we assume that $\Omega=\tilde{\Omega} \backslash \bigcup_{k=1}^{n} \omega_{k}$, where $\omega_{1}, \cdots, \omega_{n}, \tilde{\Omega}$ are simply connected domains with smooth $C^{1}$ boundaries, each $\omega_{k} \subset \tilde{\Omega}$, and the sets $\overline{\omega_{k}}$ are pair-wise disjoint.

A central role will be played by the magnetic potential $\mathbf{F} \in H^{2}(\tilde{\Omega})$ satisfying

$$
\operatorname{curl} \mathbf{F}=B, \quad \operatorname{div} \mathbf{F}=0 \quad \text { in } \tilde{\Omega}, \quad \nu \cdot \mathbf{F}=0 \quad \text { on } \partial \tilde{\Omega},
$$

where $\nu$ is the unit interior normal vector of $\partial \tilde{\Omega}$. Since the domain $\tilde{\Omega}$ is simply connected, $\mathbf{A}-\mathbf{F}$ is a gradient field on $\tilde{\Omega}$ and we can find a function $\vartheta \in H^{3}(\tilde{\Omega})$ such that (see [6, Prop. D.1.1])

$$
\mathbf{A}=\mathbf{F}+\nabla \vartheta \quad \text { on } \tilde{\Omega} .
$$

The functional \& critical configurations.

The GL functional is defined for configurations $(\psi, \mathcal{A})$ in the space $H^{1}(\Omega ; \mathbb{C}) \times H^{1}\left(\tilde{\Omega} ; \mathbb{R}^{2}\right)$ as follows

$$
\mathcal{G}(\psi, \mathcal{A})=\int_{\Omega}\left(|(\nabla-i \kappa H \mathcal{A}) \psi|^{2}-\kappa^{2}|\psi|^{2}+\frac{\kappa^{2}}{2}|\psi|^{4}\right) d x+(\kappa H)^{2} \int_{\tilde{\Omega}}|\operatorname{curl}(\mathcal{A}-\mathbf{F})|^{2} d x,
$$

where $\mathbf{F}$ is the magnetic potential introduced in (1.13). We introduce the ground state energy

$$
\mathrm{E}(\kappa, H)=\inf \left\{\mathcal{G}(\psi, \mathcal{A}):(\psi, \mathcal{A}) \in H^{1}(\Omega ; \mathbb{C}) \times H_{\text {div }}^{1}\left(\tilde{\Omega} ; \mathbb{R}^{2}\right)\right\}
$$

where $\mathcal{A} \in H_{\text {div }}^{1}\left(\tilde{\Omega} ; \mathbb{R}^{2}\right)$ means

$$
\mathcal{A} \in H^{1}\left(\tilde{\Omega} ; \mathbb{R}^{2}\right), \quad \operatorname{div} \mathcal{A}=0 \quad \text { in } \tilde{\Omega}, \quad \nu \cdot \mathcal{A}=0 \quad \text { on } \partial \tilde{\Omega},
$$

and $\nu$ is the inward normal vector of $\partial \tilde{\Omega}$. The property of gauge invariance yields [6, Sec. 10.1.2]

$$
\mathrm{E}(\kappa, H)=\inf \left\{\mathcal{G}(\psi, \mathcal{A}):(\psi, \mathcal{A}) \in H^{1}(\Omega ; \mathbb{C}) \times H^{1}\left(\tilde{\Omega} ; \mathbb{R}^{2}\right)\right\}
$$

Every minimizing configuration $(\psi, \mathcal{A})_{\kappa, H}$ is a critical point of the GL functional, that is it satisfies the following equations:

$$
\begin{cases}-(\nabla-i \kappa H \mathcal{A})^{2} \psi=\kappa^{2}\left(1-|\psi|^{2}\right) \psi & \text { in } \Omega \\ -\nabla^{\perp}(\operatorname{curl}(\mathcal{A}-\mathbf{F}))=\frac{1}{\kappa H} \mathbf{1}_{\Omega} \operatorname{Im}(\bar{\psi}(\nabla-i \kappa H \mathcal{A}) \psi) & \text { in } \tilde{\Omega} \\ \nu \cdot(\nabla-i \kappa H \mathcal{A}) \psi=0 & \text { on } \partial \Omega \\ \operatorname{curl}(\mathcal{A}-\mathbf{F})=0 & \text { on } \partial \tilde{\Omega}\end{cases}
$$

where $\nabla^{\perp}=\left(\partial_{x_{2}},-\partial_{x_{1}}\right)$ is the Hodge gradient. 
Bulk energy function.

The GL ground state energy $\mathrm{E}(\kappa, H)$ in (1.16) is closely related to a simplified effective energy, which we will call the bulk energy function. This is the concave function $g:[0,+\infty) \rightarrow\left[-\frac{1}{2}, 0\right]$ that we will introduce below. First, we set $g(0)=-\frac{1}{2}$ and $g(b)=0$ for all $b \geq 1$; the definition of $g(b)$ when $b \in(0,1)$ is implicit through the large area limit of a certain non-linear energy [1, 17, 20].

Let $R>0$ and $Q_{R}=(-R / 2, R / 2) \times(-R / 2, R / 2)$. We define the following Ginzburg-Lamdau energy with the constant magnetic field on $H^{1}\left(Q_{R}\right)$ by

$$
G_{b, Q_{R}}(u)=\int_{Q_{R}}\left(b\left|\left(\nabla-i \mathbf{A}_{\mathbf{0}}\right) u\right|^{2}-|u|^{2}+\frac{1}{2}|u|^{4}\right) d x .
$$

Here $\mathbf{A}_{\mathbf{0}}$ is the vector field introduced in (1.3). We introduce the two ground state energies

$$
m_{0}(b, R)=\inf _{u \in H_{0}^{1}\left(Q_{R}\right)} G_{b, Q_{R}}(u), \quad \text { and } \quad m(b, R)=\inf _{u \in H^{1}\left(Q_{R}\right)} G_{b, Q_{R}}(u) .
$$

We gather the following remarkable properties (see [7, Thm. 2.1]):

- If $b \geq 1$ and $R>0$, then $m_{0}(b, R)=0$.

- $m_{0}(0, R)=-\frac{R^{2}}{2}$.

- Every minimizer $u_{b, R}$ of $m_{0}(b, R)$ or $m(b, R)$ satisfies the uniform bound $\left|u_{b, R}\right| \leq 1$.

- For all $b \in[0, \infty)$, the following limits exist

$$
g(b)=\lim _{R \rightarrow \infty} \frac{m_{0}(b, R)}{R^{2}}=\lim _{R \rightarrow \infty} \frac{m(b, R)}{R^{2}} .
$$

- There exist positive constants $C$ and $R_{0}$, such that, for all $R \geq R_{0}$ and $b \in[0,1)$,

$$
g(b) \leq \frac{m_{0}(b, R)}{R^{2}} \leq g(b)+\frac{C}{R} \quad \text { and } \quad g(b)-\frac{C}{R} \leq \frac{m(b, R)}{R^{2}} \leq g(b)+\frac{C}{R}
$$

The leading order energy.

The approximation of the energy $\mathrm{E}(\kappa, H)$ will require the decomposition of the domain $\Omega$ into small cells, which we describe below and eventually define the leading order energy in (1.27).

We fix two positive constants $c_{1}$ and $c_{2}$ such that $0<c_{1}<c_{2}$, and we let $\ell$ be a parameter that varies in the following manner

$$
c_{1} \kappa^{-3 / 4} \leq \ell \leq c_{2} \kappa^{-3 / 4}
$$

so that $\ell$ approaches 0 in the regime of large GL parameter $\kappa$.

Now we set

and

$$
\begin{gathered}
x_{m, n}^{\ell}:=(\ell m, \ell n) \quad\left((m, n) \in \mathbb{Z}^{2}\right), \\
\mathcal{J}_{\ell}=\left\{x_{m, n}^{\ell}:(m, n) \in \mathbb{Z}^{2} \& Q_{\ell}\left(x_{m, n}^{\ell}\right) \subset \Omega\right\},
\end{gathered}
$$

$$
\Omega_{\ell}:=\bigcup_{x \in \mathcal{J}_{\ell}} Q_{\ell}(x)
$$

where $Q_{\ell}(\cdot)$ is the open square introduced in (2.1). The definition of the set $\mathcal{J}_{\ell}$ yields that the squares $\left(Q_{\ell}(x)\right)_{x \in \mathcal{J}_{\ell}}$ are pairwise disjoint, and $\Omega_{\ell} \subset \Omega$. Consequently the set $\mathcal{J}_{\ell}$ is finite, since the domain $\Omega$ is bounded, and its cardinal

$$
N(\ell):=\operatorname{Card}\left(\mathcal{J}_{\ell}\right)
$$

satisfies the obvious upper bound

$$
N(\ell) \leq|\Omega| \ell^{-2}
$$

Furthermore, by smoothness and boundedness of the boundary $\partial \Omega$, we can write the following lower bound on the number $N(\ell)$,

$$
N(\ell) \geq|\Omega| \ell^{-2}-\mathcal{O}\left(\ell^{-1}\right) \quad\left(\ell \rightarrow 0_{+}\right) .
$$


We demonstrate in Theorem 1.3 below that the GL ground state energy, $\mathrm{E}(\kappa, H)$, introduced in (1.16), is to leading order given by the following energy

$$
\mathrm{E}^{\mathrm{asy}}(b, \ell)=\ell^{2} \sum_{x \in \mathcal{J}_{\ell}} g\left(b B_{\mathrm{av}}^{\ell}(x)\right),
$$

where $\ell$ and $\mathcal{J}_{\ell}$ are introduced in (1.20) and (1.22) respectively, and $g(\cdot)$ is the bulk energy function introduced in (1.19).

Theorem 1.3. Assume that there exists a positive real number $c$ such that $B \geq c>0$ a.e. in $\Omega$. Given $\epsilon \in(0,1)$ and $c_{2}>c_{1}>0$, there exist constants $C, \kappa_{0}$ such that, for all $\kappa \geq \kappa_{0}, H=b \kappa, \ell$ satisfying (1.20), and $b \in\left(\epsilon, \epsilon^{-1}\right)$, the following holds

$$
\left|\mathrm{E}(\kappa, H)-\kappa^{2} \mathrm{E}^{\text {asy }}(b, \ell)\right| \leq C \kappa^{15 / 8} .
$$

Remark 1.4. Since $g(\cdot) \geq-\frac{1}{2}$, we get by (1.25),

$$
-\frac{1}{2}|\Omega| \leq \ell^{2} \sum_{x \in \mathcal{J}_{\ell}} g\left(b B_{\mathrm{av}}^{\ell}(x)\right) \leq 0 .
$$

Furthermore, since $g(\cdot)$ is concave, $-g(\cdot)$ is convex and Jensen's inequality yields

$$
\ell^{2} \sum_{x \in \mathcal{J}_{\ell}} g\left(b B_{\mathrm{av}}^{\ell}(x)\right) \geq \sum_{x \in \mathcal{J}_{\ell}} \int_{Q_{\ell}(x)} g(b B(y)) d y=\int_{\Omega} g(b B(y)) d y+\mathcal{O}(\ell) .
$$

Consequently, we see that, if $b>0$ is a fixed constant (independent from the parameters $\kappa, H, \ell$ ), the effective energy in (1.27), satisfies (see Remark 6.3 for additional details)

$$
\mathrm{E}^{\text {asy }}(b, \ell) \underset{\ell \rightarrow 0_{+}}{=} o(1) \Longleftrightarrow|\{y \in \Omega: b B(y)<1\}|=0 .
$$

Remark 1.5. We can deduce the eigenvalue upper bound mentioned in Theorem 1.2 from Theorem 1.3, by using the GL order parameter as a trial state for the Dirichlet eigenvalue. We present this construction in Sec. 6.5, which highlights the possibility of extracting spectral asymototics from the study of the GL model, despite the many existing results that go in the opposite direction, namely studying the GL model starting from eigenvalue estimates of the magnetic Laplacian.

1.5. Organization of the paper. The paper is organized as follows. Section 2 contains some standard material that we are going to use through the paper. Section 3 contains the proof of the averaging estimate, Theorem 1.1. The estimate of the energy in (1.5) occupies Section 4. The proof of Theorem 1.2 is given in Section 5 . Section 6 is devoted to the study of the GinzburgLandau model and ends up by an alternative proof of the eigenvalue upper bound for the Dirichlet magnetic Laplacian (Sec. 6.5).

\section{Preliminaries AND NOtATion}

The purpose of this section is to introduce the necessary material for the statement of the main theorems in the subsequent sections.

Asymptotic order. We will use the standard Landau notation to denote bounded quantities, $\mathcal{O}(1)$, and vanishingly small quantities, $o(1)$, with respect to a parameter $\sigma$ living in a neighborhood of $+\infty$. Additionally, we use the notation $\approx$ in the following context; given two functions $a(\sigma)$ and $b(\sigma)$, writing $a \approx b$ means that there exist positive constants $\sigma_{0}, c_{1}, c_{2}$ such that $c_{1} b(\sigma) \leq a(\sigma) \leq c_{2} b(\sigma)$. We use the letter $C$ to denote constants. The value of $C$ might change from one inequality to another without mentioning this explicitly. 
The averaged magnetic field. For all $x \in \mathbb{R}^{2}$ and $\ell>0$, we introduce the open square of center $x$ and side-length $\ell$ as follows

$$
Q_{\ell}(x)=(x-\ell / 2, x+\ell / 2) \times(x-\ell / 2, x+\ell / 2) .
$$

We introduce the averaged magnetic field in the square $Q_{\ell}(x)$,

$$
B_{\mathrm{av}}^{\ell}(x)=\frac{1}{\ell^{2}} \int_{Q_{\ell}(x)} B(y) d y .
$$

Note that, if $B$ satisfies the following condition in some open set $\Omega \subset \mathbb{R}^{2}$,

$$
\exists c \in \mathbb{R}, \quad B \geq c \quad \text { a.e. }
$$

then the averaged magnetic field satisfies

$$
B_{\mathrm{av}}^{\ell}(x) \geq c \quad \text { whenever } Q_{\ell}(x) \subset \Omega .
$$

Assuming (1.1), we will prove that $B_{\mathrm{av}}^{\ell}(x)$ can have only slow growth in the small length limit.

Lemma 2.1. For all $\zeta \in\left(0, \frac{1}{2}\right]$, there exist $C, \ell_{0}>0$ such that, for all $\ell \in\left(0, \ell_{0}\right), B \in H^{1}\left(\mathbb{R}^{2}\right)$ and $x \in \mathbb{R}^{2}$, the following holds,

$$
\left|B_{\mathrm{av}}^{\ell}(x)\right| \leq C \ell^{-2 \zeta}\|B\|_{H^{1}\left(\mathbb{R}^{2}\right)} .
$$

Proof. Notice that,

$$
\left|B_{\mathrm{av}}^{\ell}(x)\right| \leq \frac{1}{\ell^{2}} \int_{Q_{\ell}(x)}|B(y)| d y .
$$

Let $p=\frac{1}{\zeta}$ and $q=\frac{p}{p-1}$ the Hölder conjugate of $p$. By Hölder's inequality

$$
\int_{Q_{\ell}(x)}|B(y)| d y \leq\left|Q_{\ell}(x)\right|^{1 / q}\|B\|_{L^{p}\left(\mathbb{R}^{2}\right)} .
$$

Consequently,

$$
\left|B_{\mathrm{av}}^{\ell}(x)\right| \leq \ell^{\frac{2}{q}-2}\|B\|_{L^{p}\left(\mathbb{R}^{2}\right)}=\ell^{-2 \zeta}\|B\|_{L^{p}\left(\mathbb{R}^{2}\right)} .
$$

To finish the proof, we note that $p \geq 2$ and use the Sobolev embedding of $H^{1}\left(\mathbb{R}^{2}\right)$ in $L^{p}\left(\mathbb{R}^{2}\right)$.

\section{Averaging of the MAGNEtic FiELD}

The proof of Theorem 1.1 relies on the following proposition.

Proposition 3.1. For every $s \in(0,1)$, and every domain $U \subset \mathbb{R}^{2}$ satisfying (1.6), the following inequality holds,

$$
s^{2} \int_{U}\left|B_{s, x_{0}}(x)-B_{\mathrm{av}}^{U}\right|^{2} d x \leq 8 \delta^{2}\|\nabla B\|_{L^{2}(U)}^{2},
$$

where $\delta=\operatorname{diam}(U), B \in H^{1}\left(\mathbb{R}^{2}\right), B_{\mathrm{av}}^{U}$ is introduced in (1.9) and, for any $x \in U$,

$$
B_{s, x_{0}}(x):=B\left(s\left(x-x_{0}\right)+x_{0}\right) .
$$

Proof. We will prove Proposition 3.1 in the special case where $B \in C^{1}\left(\mathbb{R}^{2}\right)$. The general case follows then by a density argument, using the density of $C^{\infty}\left(\mathbb{R}^{2}\right)$ in $H^{1}\left(\mathbb{R}^{2}\right)$ and the Sobolev embedding of $H^{1}\left(\mathbb{R}^{2}\right)$ in $L^{4}\left(\mathbb{R}^{2}\right)$.

We start by noticing that

$$
B_{s, x_{0}}(x)-B_{\mathrm{av}}^{U}=\frac{1}{|U|} \int_{U}\left(B_{s, x_{0}}(x)-B(y)\right) d y .
$$

By Jensen's inequality,

$$
\int_{U}\left|B_{s, x_{0}}(x)-B_{\mathrm{av}}^{U}\right|^{2} d x \leq \frac{1}{|U|} \int_{U}\left(\int_{U}\left|B_{s, x_{0}}(x)-B(y)\right|^{2} d y\right) d x .
$$


Now, it is enough to prove the following inequality,

$$
\int_{U} \int_{U}\left|B_{s, x_{0}}(x)-B(y)\right|^{2} d x d y \leq 8 \delta^{2}|U|\|\nabla B\|_{L^{2}(U)}^{2} .
$$

Indeed, for $y, z \in U$ with $y \neq z$, the convexity of $U$ ensures that $z+t \frac{y-z}{|y-z|} \in U$ for $t \in[0,|y-z|]$, hence,

$$
\begin{aligned}
B(z)-B(y) & =-\int_{0}^{|y-z|} \frac{d}{d t} B\left(z+t \frac{y-z}{|y-z|}\right) d t \\
& =-\int_{0}^{|y-z|} \nabla B\left(z+t \frac{y-z}{|y-z|}\right) \cdot \frac{y-z}{|y-z|} d t .
\end{aligned}
$$

Consequently,

$$
\begin{aligned}
|B(z)-B(y)| & \leq \int_{0}^{|y-z|}\left|\nabla B\left(z+t \frac{y-z}{|y-z|}\right)\right| d t \\
& =|y-z| \int_{0}^{1}|\nabla B(z+\tau(y-z))| d \tau
\end{aligned}
$$

after performing the change of variable $\tau=t /|y-z|$. By Jensen's inequality, we get further

$$
\begin{aligned}
|B(z)-B(y)|^{2} & \leq|y-z|^{2} \int_{0}^{1}|\nabla B(z+\tau(y-z))|^{2} d \tau \\
& \leq \delta^{2} \int_{0}^{1}|\nabla B(z+\tau(y-z))|^{2} d \tau .
\end{aligned}
$$

We use the foregoing inequality for $z=m_{s, x_{0}}(x):=s\left(x-x_{0}\right)+x_{0}, x \in U$, from which we get

$$
\left|B_{s, x_{0}}(x)-B(y)\right|^{2} \leq \delta^{2}\left(I_{1}(x, y ; s)+I_{2}(x, y ; s)\right),
$$

where

and

$$
I_{1}(x, y ; s):=\int_{0}^{1 / 2}\left|\nabla B\left(m_{s, x_{0}}(x)+\tau\left(y-m_{s, x_{0}}(x)\right)\right)\right|^{2} d \tau
$$

$$
I_{2}(x, y ; s):=\int_{1 / 2}^{1}\left|\nabla B\left(m_{s, x_{0}}(x)+\tau\left(y-m_{s, x_{0}}(x)\right)\right)\right|^{2} d \tau .
$$

For $\tau \in[0,1]$ and $y \in U$, consider the set $U_{y, \tau}=\left\{m_{s, x_{0}}(x)+\tau\left(y-m_{s, x_{0}}(x)\right): x \in U\right\}$; since $U$ is convex, we observe that $U_{y, \tau} \subset U$. Now, performing the change of variable

$$
x \mapsto a:=m_{s, x_{0}}(x)+\tau\left(y-m_{s, x_{0}}(x)\right),
$$

we get for all $s \in(0,1)$ and $\tau \in\left[0, \frac{1}{2}\right]$,

$$
\int_{U}\left|\nabla B\left(m_{s, x_{0}}(x)+\tau\left(y-m_{s, x_{0}}(x)\right)\right)\right|^{2} d x=\frac{1}{s^{2}\left(1-\tau^{2}\right)} \int_{U_{y, \tau}}|\nabla B(a)|^{2} d a \leq \frac{4}{s^{2}}\|\nabla B\|_{L^{2}(U)}^{2} .
$$

Integrating again with respect to $y \in U$, we get

$$
\int_{U}\left(\int_{U} I_{1}(x, y ; s) d x\right) d y \leq \frac{4}{s^{2}}|U|\|\nabla B\|_{L^{2}(U)}^{2} .
$$

We estimate the integral of $I_{2}$ in a similar fashion. Doing the change of variable $y \mapsto \tilde{a}:=$ $m_{s, x_{0}}(x)+\tau\left(y-m_{s, x_{0}}(x)\right)$, we observe for all $\tau \in\left[\frac{1}{2}, 1\right]$,

$$
\int_{U}\left|\nabla B\left(m_{s, x_{0}}(x)+\tau\left(y-m_{s, x_{0}}(x)\right)\right)\right|^{2} d y=\frac{1}{\tau^{2}} \int_{V_{x, \tau}}|\nabla B(\tilde{a})|^{2} d \tilde{a} \leq 4\|\nabla B\|_{L^{2}(U)}^{2},
$$


where $V_{x, \tau}:=\left\{m_{s, x_{0}}(x)+\tau\left(y-m_{s, x_{0}}(x)\right): y \in U\right\} \subset U$, since $U$ is convex. After integrating with respect to $x \in U$, we get

$$
\int_{U}\left(\int_{U} I_{2}(x, y ; s) d y\right) d x \leq 4|U|\|\nabla B\|_{L^{2}(U)}^{2} .
$$

Inserting (3.3) and (3.4) into (3.2), we get eventually (3.1), which finishes the proof of Proposition 3.1.

Proof of Theorem 1.1. Collecting (1.7) and (1.10), we write, for all $x \in U$,

$$
\mathbf{A}_{\text {new }}^{U}(x)-\mathbf{A}_{\mathrm{av}}^{U}(x)=2 \int_{0}^{1}\left(B\left(s\left(x-x_{0}\right)+x_{0}\right)-B_{\mathrm{av}}^{U}\right) \mathbf{A}_{0}\left(s\left(x-x_{0}\right)\right) d s .
$$

Since $\left|\mathbf{A}_{0}\left(s\left(x-x_{0}\right)\right)\right| \leq \frac{1}{2} s\left|x-x_{0}\right| \leq \frac{1}{2} s \operatorname{diam}(U)$ on $U$, we get by using Jensen's inequality,

$$
\forall x \in U, \quad\left|\mathbf{A}_{\text {new }}^{U}(x)-\mathbf{A}_{\mathrm{av}}^{U}(x)\right|^{2} \leq \delta^{2} \int_{0}^{1}\left|B\left(s\left(x-x_{0}\right)+x_{0}\right)-B_{\mathrm{av}}^{U}\right|^{2} s^{2} d s .
$$

We apply Proposition 3.1 to estimate the term in the r.h.s. in (3.5). This finishes the proof of Theorem 1.1.

Remark 3.2. If we perform the change of variable $y=s\left(x-x_{0}\right)+x_{0}$ and note that $U$ is convex (which guarantees that $y \in U$, for all $x \in U$ ), we deduce from (3.5),

$$
\begin{aligned}
\int_{U}\left|\mathbf{A}_{\text {new }}^{U}(x)-\mathbf{A}_{\mathrm{av}}^{U}(x)\right|^{2} d x & \leq \delta^{2} \int_{0}^{1} \int_{U}\left|B\left(s\left(x-x_{0}\right)+x_{0}\right)-B_{\mathrm{av}}^{U}\right|^{2} s^{2} d x d s \\
& \leq \delta^{2} \int_{U}\left|B(y)-B_{\mathrm{av}}^{U}\right|^{2} d y
\end{aligned}
$$

\section{Approximation of the QUADratic FORM}

Given a bounded open set $U \subset \mathbb{R}^{2}$, a function $u \in H^{1}(U)$, a vector field $\mathbf{a} \in H^{1}\left(U ; \mathbb{R}^{2}\right)$ and a real number $\sigma$, we introduce

$$
q_{\sigma}(u, \mathbf{a} ; U)=\int_{U}|(\nabla-i \sigma \mathbf{a}) u|^{2} d x .
$$

Proposition 4.1. Given $\eta, \rho \in\left(0, \frac{1}{2}\right)$ and $0<c_{1}<c_{2}$, there exist constants $C^{\prime}, \sigma_{0}>0$ such that the following is true. If

- $\sigma \geq \sigma_{0}$;

- $U \subset \mathbb{R}^{2}$ is open and convex;

- $c_{1} \sigma^{-\rho} \leq \operatorname{diam}(U),|U|^{1 / 2} \leq c_{2} \sigma^{-\rho}$

- $u \in H^{1}(U) \cap L^{\infty}(U), B \in H^{1}\left(\mathbb{R}^{2}\right) \& \mathbf{A}$ defined by (1.2),

then there exists a function $\varphi:=\varphi^{U} \in H^{1}(U)$ such that

$$
\begin{aligned}
& \left(1-\sigma^{-\eta}\right) q_{\sigma}\left(v, \mathbf{A}_{\mathrm{av}}^{U} ; U\right)-C^{\prime} \sigma^{2-4 \rho+\eta}\|\nabla B\|_{L^{2}(U)}^{2}\|u\|_{L^{\infty}(U)}^{2} \\
& \quad \leq q_{\sigma}(u, \mathbf{A} ; U) \leq\left(1+\sigma^{-\eta}\right) q_{\sigma}\left(v, \mathbf{A}_{\mathrm{av}}^{U} ; U\right)+C^{\prime} \sigma^{2-4 \rho+\eta}\|\nabla B\|_{L^{2}(U)}^{2}\|u\|_{L^{\infty}(U)}^{2}
\end{aligned}
$$

where $\mathbf{A}_{\mathrm{av}}^{U}$ is introduced in (1.10) and $v=e^{i \sigma \varphi} u$.

Later in the proof of Theorem 1.2, we use the upper bound in Proposition 4.1 to compute the energy of a quasi-mode.

Remark 4.2. The condition $\rho \in\left(0, \frac{1}{2}\right)$ is a consequence of a scaling argument. Since $x_{0} \in U$ and $\operatorname{diam}(U) \approx \sigma^{-\rho}$, we have $U \subset\left\{\left|x-x_{0}\right| \leq \mathcal{O}\left(\sigma^{-\rho}\right)\right\}$. The change of variable, $y=\sigma^{1 / 2}\left(x-x_{0}\right)$ yields (see (1.10) $)$

$$
q_{\sigma}\left(v, \mathbf{A}_{\mathrm{av}}^{U} ; U\right)=\sigma \int_{\tilde{U}_{\sigma}}\left|\left(\nabla-B_{\mathrm{av}}^{U} \mathbf{A}_{0}\right) \tilde{v}\right|^{2} d y
$$


where $\tilde{U}_{\sigma}=\left\{y=\sigma^{1 / 2}\left(x-x_{0}\right), x \in U\right\} \subset\left\{|y| \leq \mathcal{O}\left(\sigma^{\frac{1}{2}-\rho}\right)\right\}$ and $\tilde{v}(y)=v(x)$. To ensure that $\tilde{U}_{\sigma}$ approaches $\mathbb{R}^{2}$ (which is a fixed domain), we impose the condition $\rho \in\left(0, \frac{1}{2}\right)$.

Proof of Proposition 4.1. Note that the following holds:

i. (Gauge transformation) if $v=e^{-i \sigma \phi} u$, then $q_{\sigma}\left(v, \mathbf{A}_{\mathrm{av}}^{U}, U\right)=q_{\sigma}\left(v, \mathbf{A}_{\mathrm{av}}^{U}-\nabla \phi, U\right)$;

ii. (Cauchy's inequality) for every $a, b, \sigma>0,(a+b)^{2} \leq\left(1+\sigma^{-\eta}\right) a^{2}+\left(1+\sigma^{\eta}\right) b^{2}$;

iii. Theorem 1.1;

iv. $\delta:=\operatorname{diam}(U)$ satisfies $\delta^{4} \leq c_{2}^{4} \sigma^{-4 \rho}$.

Now we write

$$
\begin{aligned}
q_{\sigma}(u, \mathbf{A} ; U) & :=\int_{U}|(\nabla-i \sigma \mathbf{A}) u|^{2} d x \\
& =\int_{U}\left|\left(\nabla-i \sigma\left(\mathbf{A}_{\mathrm{new}}^{U}-\mathbf{A}_{\mathrm{av}}^{U}+\mathbf{A}_{\mathrm{av}}^{U}-\nabla \phi\right)\right) u\right|^{2} d x \\
& \stackrel{\text { i. }}{=} \int_{U}\left|\left(\nabla-i \mathbf{A}_{\mathrm{av}}^{U}\right) v-i \sigma\left(\mathbf{A}_{\mathrm{new}}^{U}-\mathbf{A}_{\mathrm{av}}^{U}\right) u\right|^{2} d x \\
& \stackrel{\text { ii. }}{\leq}\left(1+\sigma^{-\eta}\right) \int_{U}\left|\left(\nabla-i \sigma \mathbf{A}_{\mathrm{av}}^{U}\right) v\right|^{2} d x+\left(1+\sigma^{\eta}\right) \sigma^{2} \int_{U}\left|\left(\mathbf{A}_{\mathrm{new}}^{U}-\mathbf{A}_{\mathrm{av}}^{U}\right) u\right|^{2} d x \\
& \text { iii. }\left(1+\sigma^{-\eta}\right) \int_{U}\left|\left(\nabla-i \sigma \mathbf{A}_{\mathrm{av}}^{U}\right) v\right|^{2} d x+8 \sigma^{2}\left(1+\sigma^{\eta}\right) \delta^{4}\|\nabla B\|_{L^{2}(U)}^{2}\|u\|_{L^{\infty}(U)}^{2} \\
& \leq\left(1+\sigma^{-\eta}\right) q_{\sigma}\left(v, \mathbf{A}_{\mathrm{av}}^{U} ; U\right)+8 \sigma^{2}\left(1+\sigma^{\eta}\right) c_{2}^{4} \sigma^{-4 \rho}\|\nabla B\|_{L^{2}(U)}^{2}\|u\|_{L^{\infty}(U)}^{2} \\
& \leq\left(1+\sigma^{-\eta}\right) q_{\sigma}\left(v, \mathbf{A}_{\mathrm{av}}^{U} ; U\right)+C^{\prime} \sigma^{2-4 \rho+\eta}\|\nabla B\|_{L^{2}(U)}^{2}\|u\|_{L^{\infty}(U)}^{2}, \\
& \leq\left(1+\sigma^{-}\right.
\end{aligned}
$$

with $C^{\prime}=16 c_{2}^{4}$. A similar argument yields

$$
\left(1-\sigma^{-\eta}\right) q_{\sigma}\left(v, \mathbf{A}_{\mathrm{av}}^{U} ; U\right)-C^{\prime} \sigma^{2-4 \rho+\eta}\|\nabla B\|_{L^{2}(U)}^{2}\|u\|_{L^{\infty}(U)}^{2} \leq q_{\sigma}(u, \mathbf{A} ; U) .
$$

\section{Magnetic Laplacian}

The aim of this section is to prove Theorem 1.2, which is concerned with the principal eigenvalue of the magnetic Laplacian

$$
\Delta_{\sigma \mathbf{A}}=-(\nabla-i \sigma \mathbf{A})^{2}
$$

with domain (when $\Omega \subset \mathbb{R}^{2}$ is bounded and with a smooth $C^{2}$ boundary)

$$
\mathcal{D}=H^{2}(\Omega) \cap H_{0}^{1}(\Omega) .
$$

The operator $\Delta_{\sigma \mathbf{A}}$ is self-adjoint in the Hilbert space $L^{2}(\Omega)$ and its principal eigenvalue is introduced in (1.12).

5.1. Upper bound. We will construct a trial state by means of a Gaussian function, but localized near a point $x_{\varepsilon} \in \Omega$ such that the Lebesgue differentiation theorem holds for $B(x)$ and $|\nabla B(x)|$ at $x_{\varepsilon}$, and as $\varepsilon \rightarrow 0_{+}, B\left(x_{\varepsilon}\right)=m_{0}(B ; \Omega)+\mathcal{O}(\varepsilon)$, where $m_{0}(B ; \Omega)$ is the essential infimum introduced in (1.11).

By the Lebesgue differentiation theorem, the two sets

$$
\begin{aligned}
& N=\left\{u \in \Omega, \lim _{\ell \rightarrow 0+} \frac{1}{|D(u, \ell)|} \int_{D(u, \ell)}|\nabla B(x)|^{2} d x \neq|\nabla B(u)|^{2}\right\} \\
& \tilde{N}=\left\{u \in \Omega, \lim _{\ell \rightarrow 0+} \frac{1}{|D(u, \ell)|} \int_{D(u, \ell)} B(x) d x \neq B(u)\right\}
\end{aligned}
$$

have zero Lebesgue measure, where $D(u, \ell)$ denotes the open disk of center $u$ and radius $\ell$.

We assume that $m_{0}(B ; \Omega)>0$. For all $\varepsilon \in(0,1]$, we introduce the set

$$
M_{\varepsilon}=\left\{x \in \Omega, m_{0}(B ; \Omega) \leq B(x) \leq m_{0}(B ; \Omega)+\varepsilon\right\} .
$$


Since the set $M_{\varepsilon}$ has a non-zero Lebesgue measure, $M_{\varepsilon} \not \subset N \cup \tilde{N}$, so we get by the Lebesgue differentiation theorem

$$
\begin{aligned}
\exists x_{\varepsilon} \in M_{\varepsilon}, \quad \frac{1}{\left|D\left(x_{\varepsilon}, \ell\right)\right|} \int_{D\left(x_{\varepsilon}, \ell\right)}|\nabla B(x)|^{2} d x \underset{\ell \rightarrow 0_{+}}{\longrightarrow}\left|\nabla B\left(x_{\varepsilon}\right)\right|^{2}<+\infty \\
\text { and } \frac{1}{\left|D\left(x_{\varepsilon}, \ell\right)\right|} \int_{D\left(x_{\varepsilon}, \ell\right)} B(x) d x \underset{\ell \rightarrow 0_{+}}{\longrightarrow} B\left(x_{\varepsilon}\right)<+\infty .
\end{aligned}
$$

In the sequel, $\rho \in\left(0, \frac{1}{2}\right)$ and $U:=D\left(x_{\varepsilon}, \sigma^{-\rho}\right) \subset \Omega$ for $\sigma$ sufficiently large. Let $\varphi:=\varphi^{U}$ be the gauge function in Proposition 4.1. Consider the trial state $u(x)=e^{-i \sigma \varphi} v(x)$, with $v$ the following Gaussian,

$$
v(x)=\pi^{-1 / 2}\left(B_{\mathrm{av}}^{U}\right)^{1 / 4} \sigma^{1 / 2} \chi\left(\sigma^{\rho}\left(x-x_{\varepsilon}\right)\right) \exp \left(-\frac{1}{2}\left(B_{\mathrm{av}}^{U}\right)^{1 / 2} \sigma\left|x-x_{\varepsilon}\right|^{2}\right),
$$

where $\chi \in C_{c}^{\infty}\left(\mathbb{R}^{2} ;[0,1]\right)$ is supported in the unit disk and equal to 1 on $\left\{|x| \leq \frac{1}{2}\right\}$. By a change of variable, we see that

and

$$
\|u\|_{L^{2}(U)}^{2}=\|v\|_{L^{2}(U)}^{2}=1+o\left(\sigma^{-1}\right)
$$

$$
q_{\sigma}\left(v, \mathbf{A}_{\mathrm{av}}^{U} ; U\right)=B_{\mathrm{av}}^{U} \sigma+o(\sigma) .
$$

Note that $\|u\|_{L^{\infty}(U)}^{2}=\pi^{-1}\left(B_{\mathrm{av}}^{U}\right)^{1 / 2} \sigma=\mathcal{O}(\sigma)$ by (5.3). We deduce from Proposition 4.1 ,

$$
\frac{q_{\sigma}(u, \mathbf{A} ; U)}{\|u\|_{L^{2}(U)}^{2}} \leq\left(1+\sigma^{-\eta}\right) \sigma B_{\mathrm{av}}^{U}+\mathcal{O}\left(\|\nabla B\|_{L^{2}(U)}^{2} \sigma^{3-4 \rho+\eta}\right) .
$$

Let us choose $\rho=3 / 8$ and $\eta=1 / 8$. Since $U=D\left(x_{\varepsilon}, \sigma^{-\rho}\right)$, the error term can be expressed in the following pleasant form

$$
\|\nabla B\|_{L^{2}(U)}^{2} \sigma^{3-4 \rho+\eta}=\frac{1}{|U|}\|\nabla B\|_{L^{2}(U)}^{2} \pi \sigma^{3-6 \rho+\eta}=\frac{1}{|U|}\|\nabla B\|_{L^{2}(U)}^{2} \pi \sigma^{7 / 8} .
$$

So we infer from (5.3) that

$$
\frac{q_{\sigma}(u, \mathbf{A} ; U)}{\|u\|_{L^{2}(U)}^{2}} \leq\left(1+\sigma^{-\eta}\right) \sigma B\left(x_{\varepsilon}\right)+o(\sigma) .
$$

Since $u$ is supported in $U$, we deduce from the min-max principle (1.12),

$$
\lambda(\sigma, \mathbf{A} ; \Omega) \leq B\left(x_{\varepsilon}\right) \sigma+o(\sigma) \stackrel{\left(x_{\varepsilon} \in M_{\varepsilon}\right)}{\leq}\left(m_{0}(B ; \Omega)+\varepsilon\right) \sigma+o(\sigma) .
$$

Taking the successive limits, as $\sigma \rightarrow+\infty$ then as $\varepsilon \rightarrow 0_{+}$, we get

$$
\limsup _{\sigma \rightarrow+\infty} \frac{\lambda(\sigma, \mathbf{A} ; \Omega)}{\sigma} \leq m_{0}(B ; \Omega) \text {. }
$$

5.2. Lower bound. The lower bound in Theorem 1.2 is non-asymptotic and does not require the hypothesis that the essential infimum is strictly positive.

Proposition 5.1. Let $\mathbf{A} \in H^{1}\left(\mathbb{R}^{2} ; \mathbb{R}^{2}\right)$ and $B=\operatorname{curl} \mathbf{A}$. For all $u \in C_{c}^{\infty}(\Omega)$ and $\sigma>0$, the following lower bound holds

$$
\int_{\Omega}|(\nabla-i \sigma \mathbf{A}) u|^{2} d x \geq \sigma \int_{\Omega} B(x)|u(x)|^{2} d x .
$$

Proof. Consider a sequence $\left(\mathbf{A}_{n}\right)_{n \geq 1} \subset C^{\infty}\left(\mathbb{R}^{2} ; \mathbb{R}^{2}\right)$ such that $\mathbf{A}_{n} \rightarrow \mathbf{A}$ in $H^{1}\left(\mathbb{R}^{2} ; \mathbb{R}^{2}\right)$. For all $n \geq 1$, let $B_{n}=$ curl $\mathbf{A}_{n}$. Note that $B_{n} \rightarrow B$ in $L^{2}\left(\mathbb{R}^{2}\right)$.

Fix $u \in C_{c}^{\infty}(\Omega)$. Since $\mathbf{A}_{n}$ is smooth, we have (see [6, Lem. 1.4.1])

$$
\int_{\Omega}\left|\left(\nabla-i \sigma \mathbf{A}_{n}\right) u\right|^{2} d x \geq \sigma \int_{\Omega} B_{n}(x)|u(x)|^{2} d x .
$$


It is easy to check that

$$
\begin{aligned}
\lim _{n \rightarrow+\infty} \int_{\Omega}\left|\left(\nabla-i \sigma \mathbf{A}_{n}\right) u\right|^{2} d x=\int_{\Omega}|(\nabla-i \sigma \mathbf{A}) u|^{2} d x \\
\quad \text { and } \lim _{n \rightarrow+\infty} \int_{\Omega} B_{n}(x)|u(x)|^{2} d x=\int_{\Omega} B(x)|u(x)|^{2} d x .
\end{aligned}
$$

In fact,

$$
\left|\left\|\left(\nabla-i \sigma \mathbf{A}_{n}\right) u\right\|_{L^{2}(\Omega)}-\|(\nabla-i \sigma \mathbf{A}) u\|_{L^{2}(\Omega)}\right| \leq \sigma\left\|\mathbf{A}_{n}-\mathbf{A}\right\|_{L^{4}(\Omega)}\|u\|_{L^{4}(\Omega)}
$$

and

$$
\left.\left|\int_{\Omega}\left(B_{n}(x)-B(x)\right)\right| u(x)\right|^{2} d x \mid \leq\left\|B_{n}-B\right\|_{L^{2}(\Omega)}\|u\|_{L^{4}(\Omega)}^{2} .
$$

5.3. Proof of Theorem 1.2. Collect (5.4) and Proposition 5.1.

6. The Ginzburg-Landau model

This section is devoted to the proof of Theorem 1.3. Also, in Sec. 6.5, we use Theorem 1.3 to give a new proof of Theorem 1.2 .

6.1. Lower bound of GL energy. In the sequel, $(\psi, \mathcal{A})_{\kappa, H}$ denotes a configuration in the space $H^{1}(\Omega ; \mathbb{C}) \times H^{1}\left(\Omega ; \mathbb{R}^{2}\right)$ such that

$$
\mathcal{G}(\psi, \mathcal{A})=\mathrm{E}(\kappa, H)
$$

Our aim is to prove the following proposition.

Proposition 6.1. Given $\epsilon \in(0,1)$, there exist $C, \kappa_{0}>0$ such that the following inequality holds

$$
\mathcal{G}_{0}\left(\psi, \mathcal{A} ; Q_{\ell}\left(x_{0}\right)\right) \geq g\left(b B_{\mathrm{av}}^{\ell}\left(x_{0}\right)\right) \kappa^{2} \ell^{2}-C\left(\kappa^{15 / 8} \ell^{2}+\kappa^{3 / 2}\|\nabla B\|_{L^{2}\left(Q_{\ell}\left(x_{0}\right)\right.}^{2}\right),
$$

where

- $x_{0} \in \mathcal{J}_{\ell}$

- $\ell=\kappa^{-3 / 4}$;

- $(\psi, \mathcal{A})_{\kappa, H}$ is a minimizer of the GL functional;

- $H=b \kappa$ and $b \in\left(\epsilon, \frac{1}{\epsilon}\right)$;

- $\mathcal{G}_{0}\left(\psi, \mathcal{A} ; Q_{\ell}\left(x_{0}\right)\right)=\int_{Q_{\ell}\left(x_{0}\right)}\left(|(\nabla-i \kappa H \mathcal{A}) \psi|^{2}-\kappa^{2}|\psi|^{2}+\frac{\kappa^{2}}{2}|\psi|^{4}\right) d x$.

Proof. First we notice the useful inequalities (see [4, Prop. 4.1\& Thm. 4.2])

$$
\|\psi\|_{L^{\infty}(\Omega)} \leq 1, \quad\|(\nabla-i \kappa H \mathcal{A}) \psi\|_{L^{2}(\Omega)} \leq|\Omega| \kappa, \quad\|\mathcal{A}-\mathbf{F}\|_{C^{0, \alpha}(\bar{\Omega})} \leq \frac{C_{\alpha}}{\kappa},
$$

where $\alpha \in(0,1)$ can be chosen in an arbitrary manner.

We set

$$
\phi_{x_{0}}:=\left(\mathcal{A}\left(x_{0}\right)-\mathbf{F}\left(x_{0}\right)\right) \cdot\left(x-x_{0}\right) \quad \text { and } \quad \mathcal{A}^{\text {new }}=\mathcal{A}-\nabla \phi_{x_{0}} .
$$

It is easy to check that

$$
\mathcal{G}_{0}\left(\psi, \mathcal{A} ; Q_{\ell}\left(x_{0}\right)\right)=\mathcal{G}_{0}\left(u, \mathcal{A}^{\text {new }} ; Q_{\ell}\left(x_{0}\right)\right)
$$

where

$$
u(x)=e^{-i \kappa H \phi_{x_{0}}} \psi(x) .
$$

Writing $\mathcal{A}^{\text {new }}=\mathbf{F}+\mathcal{A}^{\text {new }}-\mathbf{F}$, we get by Cauchy's inequality,

$$
\left|\left(\nabla-i \kappa H \mathcal{A}^{\text {new }}\right) u\right|^{2} \geq\left(1-\kappa^{-1 / 2}\right)|(\nabla-i \kappa H \mathbf{F}) u|^{2}-\kappa^{1 / 2}(\kappa H)^{2}\left|\left(\mathcal{A}^{\text {new }}-\mathbf{F}\right) u\right|^{2} .
$$


Consequently, we infer from the foregoing inequality and the third inequality in (6.1),

$$
\begin{array}{r}
\mathcal{G}_{0}\left(u, \mathcal{A}^{\text {new }} ; Q_{\ell}\left(x_{0}\right)\right) \geq\left(1-\kappa^{-1 / 2}\right) \mathcal{G}_{0}\left(u, \mathbf{F}, Q_{\ell}\left(x_{0}\right)\right)+\kappa^{1 / 2} \int_{Q_{\ell}\left(x_{0}\right)}\left(-\kappa^{2}|u|^{2}+\frac{\kappa^{2}}{2}|u|^{4}\right) d x \\
-C b^{2} \ell^{2 \alpha} \kappa^{5 / 2} \int_{Q_{\ell}\left(x_{0}\right)}|u|^{2} d x
\end{array}
$$

Using that $|u|=|\psi| \leq 1$ by (6.1), we can estimate the remainder terms in (6.5) as follows

$$
\begin{aligned}
\kappa^{-1 / 2} \int_{Q_{\ell}\left(x_{0}\right)}\left(-\kappa^{2}|u|^{2}+\frac{\kappa^{2}}{2}|u|^{4}\right) d x-C b^{2} \ell^{2 \alpha} \kappa^{5 / 2} \int_{Q_{\ell}\left(x_{0}\right)} & |u|^{2} d x \\
& \geq-\kappa^{2} \ell^{2}\left(\kappa^{-1 / 2}+C b^{2} \kappa^{1 / 2} \ell^{2 \alpha}\right) .
\end{aligned}
$$

In order to estimate the term $\mathcal{G}_{0}\left(u, \mathbf{F}, Q_{\ell}\left(x_{0}\right)\right)$ in (6.5), we will go from the potential $\mathbf{F}$ to the potential $\mathbf{A}$ introduced in (1.2). Let $\vartheta$ be the function in (1.14) and set

$$
v=e^{-i \kappa H \vartheta} u \text {. }
$$

Then

$$
\begin{aligned}
\mathcal{G}_{0}\left(u, \mathbf{F} ; Q_{\ell}\left(x_{0}\right)\right)= & \mathcal{G}_{0}\left(v, \mathbf{A} ; Q_{\ell}\left(x_{0}\right)\right) \\
\geq\left(1-\kappa^{-1 / 2}\right) \mathcal{G}_{0}\left(w, \mathbf{A}_{\mathrm{av}}^{Q_{\ell}\left(x_{0}\right)} ; Q_{\ell}\left(x_{0}\right)\right)-\hat{C} \kappa^{3 / 2}\|\nabla B\|_{L^{2}\left(Q_{\ell}\left(x_{0}\right)\right)}^{2} & \\
& +\kappa^{-1 / 2} \int_{Q_{\ell}\left(x_{0}\right)}\left(-\kappa^{2}|w|^{2}+\frac{\kappa^{2}}{2}|w|^{4}\right) d x
\end{aligned}
$$

where we used Proposition 4.1, with $\sigma=\kappa H=b \kappa^{2}$ and $\eta=1 / 4$, to estimate the $L^{2}$-norm of $|(\nabla-i \kappa H \mathbf{A}) v|$; the function $w$ is expressed in terms of $v$ and the gauge function $\varphi^{Q_{\ell}\left(x_{0}\right)}$ of Proposition 4.1 as follows

$$
w(x)=v(x) \exp \left(i \kappa H \varphi^{Q_{\ell}\left(x_{0}\right)}(x)\right) .
$$

Since $|v|=|u|=|\psi| \leq 1$ by (6.1), we infer from (6.8),

$$
\mathcal{G}_{0}\left(u, \mathbf{F} ; Q_{\ell}\left(x_{0}\right)\right) \geq\left(1-\kappa^{-1 / 2}\right) \mathcal{G}_{0}\left(w, \mathbf{A}_{\mathrm{av}}^{Q_{\ell}\left(x_{0}\right)} ; Q_{\ell}\left(x_{0}\right)\right)-\hat{C} \kappa^{3 / 2}\|\nabla B\|_{L^{2}\left(Q_{\ell}\left(x_{0}\right)\right)}^{2}-\kappa^{3 / 2} \ell^{2} .
$$

Note that curl $\mathbf{A}_{\mathrm{av}}^{Q_{\ell}\left(x_{0}\right)}=B_{\mathrm{av}}^{\ell}\left(x_{0}\right)$ introduced in (1.9). We write now a lower bound of the energy $\mathcal{G}_{0}\left(w, \mathbf{A}_{\mathrm{av}}^{Q_{\ell}\left(x_{0}\right)} ; Q_{\ell}\left(x_{0}\right)\right)$ using the bulk energy function $g(\cdot)$. To that end, we introduce

- $\hat{b}=\frac{H}{\kappa} B_{\mathrm{av}}^{\ell}\left(x_{0}\right)=b B_{\mathrm{av}}^{\ell}\left(x_{0}\right)$;

- $R=\ell \sqrt{\kappa H B_{\mathrm{av}}^{\ell}\left(x_{0}\right)}$;

- $h(x)=w\left(\frac{\ell}{R} x+x_{0}\right)$ for $x \in Q_{R}:=(-R / 2, R / 2)^{2}$;

- The change of variable $y=\frac{R}{\ell}\left(x-x_{0}\right)$.

It is then easy to check that

$$
\mathcal{G}_{0}\left(w, \mathbf{A}_{\mathrm{av}}^{Q_{\ell}\left(x_{0}\right)} ; Q_{\ell}\left(x_{0}\right)\right)=\frac{1}{\hat{b}} G_{\hat{b}, Q_{R}}(h) \geq m(\hat{b}, R) \geq \frac{1}{\hat{b}}\left(g(\hat{b}) R^{2}-\tilde{C} R\right),
$$

by (1.19). Inserting the foregoing inequality into (6.10), then remembering the definition of $\hat{b}$, choosing $\alpha=\frac{5}{6}$, and collecting the inequalities in (6.8), (6.6), (6.5), and (6.3), we eventually get the following inequality,

$$
\begin{aligned}
\mathcal{G}_{0}(\psi, \mathcal{A} & \left.; Q_{\ell}\left(x_{0}\right)\right) \\
& \geq g\left(b B_{\mathrm{av}}^{\ell}\left(x_{0}\right)\right) \kappa^{2} \ell^{2}-\check{C}\left(\kappa^{2} \ell^{2}\left(\kappa^{-1 / 3}+\kappa^{-1 / 4} \sqrt{B_{\mathrm{av}}^{\ell}\left(x_{0}\right)}\right)-\kappa^{3 / 2}\|\nabla B\|_{L^{2}\left(Q_{\ell}\left(x_{0}\right)\right)}^{2}\right) .
\end{aligned}
$$

Finally, we apply Lemma 2.1 with $\zeta=\frac{1}{16}$. 


\subsection{Upper bound of GL energy.}

Proposition 6.2. Given $\epsilon \in(0,1)$ and $c_{2}>c_{1}>0$, there exist $C, \kappa_{0}>0$ such that, for all $\kappa \geq \kappa_{0}$, the following holds.

For every $x_{0} \in \mathcal{J}_{\ell}$, with $\ell$ satisfying (1.20), there exists a function $v_{x_{0}, \ell} \in H_{0}^{1}\left(Q_{\ell}\left(x_{0}\right)\right)$ such that

$$
\mathcal{G}_{0}\left(v_{x_{0}, \ell}, \mathbf{F} ; Q_{\ell}\left(x_{0}\right)\right) \leq g\left(b B_{\mathrm{av}}^{\ell}\left(x_{0}\right)\right) \kappa^{2} \ell^{2}+C\left(\kappa^{7 / 4} \ell^{2}+\kappa^{3 / 2}\|\nabla B\|_{L^{2}\left(Q_{\ell}\left(x_{0}\right)\right.}^{2}\right),
$$

where

- $\mathbf{F}$ is the magnetic potential introduced in (1.13);

- $H=b \kappa$ and $b \in\left(\epsilon, \frac{1}{\epsilon}\right)$;

- the functional $\mathcal{G}_{0}\left(\cdot, \cdot ; Q_{\ell}\left(x_{0}\right)\right)$ is introduced in Proposition 6.1 .

Proof. We choose $b \in\left(\epsilon, \epsilon^{-1}\right)$ and an arbitrary point $x_{0} \in \mathcal{J}_{\ell}$, with $\ell \approx \kappa^{-3 / 4}$. We introduce the two parameters (that depend on $x_{0}$ and $\ell$ )

$$
\hat{b}=\frac{H}{\kappa} B_{\mathrm{av}}^{\ell}\left(x_{0}\right)=b B_{\mathrm{av}}^{\ell}\left(x_{0}\right) \quad \text { and } \quad R=\ell \sqrt{\kappa H B_{\mathrm{av}}^{\ell}\left(x_{0}\right)} .
$$

Let $u_{\hat{b}, R} \in H_{0}^{1}\left((-R / 2, R / 2)^{2}\right)$ be a minimizer of the energy functional $m_{0}(\hat{b}, R)$. For all $x \in$ $Q_{\ell}\left(x_{0}\right)$, we introduce the function $v:=v_{x_{0}, \ell} \in H_{0}^{1}\left(Q_{\ell}\left(x_{0}\right)\right)$ as follows

$$
v(x)=\exp (i \kappa H(\varphi+\vartheta)) u_{\hat{b}, R}\left(\frac{R}{\ell}\left(x-x_{0}\right)\right),
$$

where $\vartheta$ is the function introduced in (1.14) and $\varphi:=\varphi^{Q_{\ell}\left(x_{0}\right)}$ is the function introduced in Proposition 4.1. Setting $h=\exp (-i \kappa H(\varphi+\vartheta)) v$, it is easy to check that

$$
\mathcal{G}_{0}\left(h, \mathbf{A}_{\mathrm{av}}^{Q_{\ell}\left(x_{0}\right)} ; Q_{\ell}\left(x_{0}\right)\right)=\frac{1}{\hat{b}} m_{0}(\hat{b}, R) .
$$

Using (1.19), we get further

$$
\mathcal{G}_{0}\left(h, \mathbf{A}_{\mathrm{av}}^{Q_{\ell}\left(x_{0}\right)} ; Q_{\ell}\left(x_{0}\right)\right) \leq g\left(b B_{\mathrm{av}}^{\ell}\left(x_{0}\right)\right) \kappa^{2} \ell^{2}+\mathcal{O}(\kappa \ell) .
$$

Setting $u=\exp (-i \kappa H \vartheta) v$, we get by (1.14),

$$
\mathcal{G}_{0}\left(v, \mathbf{F} ; Q_{\ell}\left(x_{0}\right)\right)=\mathcal{G}_{0}\left(u, \mathbf{A} ; Q_{\ell}\left(x_{0}\right)\right) .
$$

Now we apply Proposition 4.1 with $\sigma=\kappa H=b \kappa^{2}, \rho=3 / 8$ and $\eta=1 / 4$; eventually we get

$$
\begin{aligned}
\mathcal{G}_{0}\left(u, \mathbf{A} ; Q_{\ell}\left(x_{0}\right)\right) & \leq\left(1+\kappa^{-1 / 2}\right) \mathcal{G}_{0}\left(h, \mathbf{A}_{\mathrm{av}}^{Q_{\ell}\left(x_{0}\right)} ; Q_{\ell}\left(x_{0}\right)\right) \\
& -\kappa^{-1 / 2} \int_{Q_{\ell}\left(x_{0}\right)}\left(-\kappa^{2}|h|^{2}+\frac{\kappa^{2}}{2}|h|^{4}\right) d x+\mathcal{O}\left(\kappa^{3}\|\nabla B\|_{L^{2}\left(Q_{\ell}\left(x_{0}\right)\right.}^{2}\right) \int_{Q_{\ell}\left(x_{0}\right)}|h|^{2} d x .
\end{aligned}
$$

Since $|h| \leq 1$, we get further

$$
\mathcal{G}_{0}\left(u, \mathbf{A} ; Q_{\ell}\left(x_{0}\right)\right) \leq\left(1+\kappa^{-1 / 2}\right) \mathcal{G}_{0}\left(h, \mathbf{A}_{\mathrm{av}}^{Q_{\ell}\left(x_{0}\right)} ; Q_{\ell}\left(x_{0}\right)\right)+\mathcal{O}\left(\kappa^{3 / 2} \ell^{2}\right)+\mathcal{O}\left(\kappa^{3 / 2}\right)\|\nabla B\|_{L^{2}\left(Q_{\ell}\left(x_{0}\right)\right)}^{2} .
$$

Collecting (6.13), (6.12) and (6.11), we finish the proof of Proposition 6.2

\subsection{Proof of Theorem $\mathbf{1 . 3}$.}

Now we work under the assumptions of Theorem 1.3. We fix $\epsilon \in(0,1)$ and assume that $H=b \kappa$ with $b$ varying in $\left(\epsilon, \epsilon^{-1}\right)$. Recall that $\ell \approx \kappa^{-3 / 4}$ by (1.20).

\section{Step 1:}

Denote by $(\psi, \mathcal{A})_{\kappa, H}$ a minimizing configuration such that $\mathcal{G}(\psi, \mathcal{A})=\mathrm{E}(\kappa, H)$. Dropping the term $\kappa^{2} H^{2} \int_{\Omega}|\operatorname{curl}(\mathcal{A}-\mathbf{F})|^{2} d x$ from the energy $\mathcal{G}(\psi, \mathcal{A})$, we get the obvious lower bound

$$
\mathrm{E}(\kappa, H)=\mathcal{G}(\psi, \mathcal{A}) \geq \mathcal{G}_{0}(\psi, \mathcal{A} ; \Omega)=\mathcal{G}_{0}\left(\psi, \mathcal{A} ; \Omega_{\ell}\right)+\mathcal{G}_{0}\left(\psi, \mathcal{A} ; \Omega \backslash \Omega_{\ell}\right)
$$


where $\mathcal{G}_{0}$ is the energy introduced in Proposition 6.1, and $\Omega_{\ell}$ is the domain introduced in (1.23). Using the uniform bounds $|\psi| \leq 1$ and $\left|\Omega \backslash \Omega_{\ell}\right|=\mathcal{O}(\ell)$, we get

$$
\mathcal{G}_{0}\left(\psi, \mathcal{A} ; \Omega \backslash \Omega_{\ell}\right) \geq-\kappa^{2} \int_{\Omega \backslash \Omega_{\ell}}=\mathcal{O}\left(\ell \kappa^{2}\right)=\mathcal{O}\left(\kappa^{5 / 4}\right) .
$$

Now, we use the obvious decomposition $\mathcal{G}_{0}\left(\psi, \mathcal{A} ; \Omega_{\ell}\right)=\sum_{x \in \mathcal{J}_{\ell}} \mathcal{G}_{0}\left(\psi, \mathcal{A} ; Q_{\ell}(x)\right)$ and apply Proposition 6.1. Eventually, we get

$$
\mathrm{E}(\kappa, H) \geq \kappa^{2} \ell^{2} \sum_{x \in \mathcal{J}_{\ell}} g\left(b B_{\mathrm{av}}^{\ell}\left(x_{0}\right)\right)-C \kappa^{5 / 4}-C \sum_{x \in \mathcal{J}_{\ell}}\left(\kappa^{15 / 8} \ell^{2}+\kappa^{3 / 2}\|\nabla B\|_{L^{2}\left(Q_{\ell}\left(x_{0}\right)\right)}^{2}\right) .
$$

Since the squares $\left(Q_{\ell}(x)\right)_{x \in \mathcal{J}_{\ell}}$ are pairwise disjoint, $\sum_{x \in \mathcal{J}_{\ell}}\|\nabla B\|_{L^{2}\left(Q_{\ell}\left(x_{0}\right)\right)}^{2}=\|\nabla B\|_{L^{2}\left(\Omega_{\ell}\right)}^{2} \leq\|\nabla B\|_{L^{2}(\Omega)}^{2}$. Using (1.25), $\sum_{x \in \mathcal{J}_{\ell}} \ell^{2}=N(\ell) \ell^{2}=\mathcal{O}(1)$. Consequently,

$$
\mathrm{E}(\kappa, H) \geq \kappa^{2} \ell^{2} \sum_{x \in \mathcal{J}_{\ell}} g\left(b B_{\mathrm{av}}^{\ell}\left(x_{0}\right)\right)+\mathcal{O}\left(\kappa^{15 / 8}\right) \quad(\kappa \rightarrow+\infty) .
$$

\section{Step 2:}

We introduce the function $\psi^{\text {trial }} \in H_{0}^{1}(\Omega)$ as follows

$$
\psi^{\text {trial }}(y)=\sum_{x \in \mathcal{J}_{\ell}} \mathbf{1}_{Q_{\ell}(x)} v_{x, \ell}(y) \quad(y \in \Omega),
$$

where, for $x \in \mathcal{J}_{\ell}, v_{x, \ell} \in H_{0}^{1}\left(Q_{\ell}(x)\right)$ is the function introduced in Proposition 6.2 and extended by 0 on $\Omega \backslash Q_{\ell}(x)$. Clearly, $\mathrm{E}(\kappa, H) \leq \mathcal{G}\left(\psi^{\text {trial }}, \mathbf{F}\right)=\mathcal{G}_{0}\left(\psi^{\text {trial }}, \mathbf{F} ; \Omega\right)$. Using Proposition 6.2 and that the squares $\left(Q_{\ell}(x)\right)_{x \in \mathcal{J}_{\ell}}$ are pairwise disjoint, we write

$$
\begin{aligned}
\mathcal{G}_{0}\left(\psi^{\text {trial }}, \mathbf{F}\right) & =\sum_{x \in \mathcal{J}_{\ell}} \mathcal{G}_{0}\left(v_{x, \ell}, \mathbf{F} ; Q_{\ell}(x)\right) \\
& \leq \kappa^{2} \ell^{2} \sum_{x \in \mathcal{J}_{\ell}} g\left(b B_{\mathrm{av}}^{\ell}(x)\right)+C \sum_{x \in \mathcal{J}_{\ell}}\left(\kappa^{7 / 4} \ell^{2}+\kappa^{3 / 2}\|\nabla B\|_{L^{2}\left(Q_{\ell}(x)\right)}^{2}\right) \\
& \leq \kappa^{2} \ell^{2} \sum_{x \in \mathcal{J}_{\ell}} g\left(b B_{\mathrm{av}}^{\ell}(x)\right)+C N(\ell) \kappa^{7 / 4} \ell^{2}+\kappa^{3 / 2}\|\nabla B\|_{L^{2}(\Omega)} \\
& =\kappa^{2} \ell^{2} \sum_{x \in \mathcal{J}_{\ell}} g\left(b B_{\mathrm{av}}^{\ell}(x)\right)+\mathcal{O}\left(\kappa^{7 / 4}\right) .
\end{aligned}
$$

6.4. Further remarks. We collect here some additional properties for later use. In the sequel, $(\psi, \mathcal{A})_{\kappa, H}$ denotes a minimizing configuration of the energy in (1.15).

We start by a rough estimate of $\mathcal{A}-\mathbf{F}$. By dropping the positive terms in the inequality $\mathcal{G}(\psi, \mathcal{A}) \leq \mathcal{G}(0, \mathbf{F})=0$ we get the following estimate

$$
\|\operatorname{curl}(\mathcal{A}-\mathbf{F})\|_{L^{2}(\tilde{\Omega})} \leq H^{-1}\|\psi\|_{L^{2}(\Omega)} .
$$

Also, $(\psi, \mathcal{A})$ being a critical point of the GL energy (see (1.18) $)$, we know that $\operatorname{curl}(\mathcal{A}-\mathbf{F})=0$ on $\partial \tilde{\Omega}$ (see [6, Eq. (10.8b)]); hence, the curl-div inequality [6, Prop. D.2.1] yields that $\mathcal{A}-\mathbf{F} \in H^{1}(\tilde{\Omega})$; we deduce then by the Sobolev embedding of $H^{1}(\tilde{\Omega})$ in $L^{4}(\tilde{\Omega})$ that

$$
\|\mathcal{A}-\mathbf{F}\|_{L^{4}(\tilde{\Omega})} \leq C_{*}\|\operatorname{curl}(\mathcal{A}-\mathbf{F})\|_{L^{2}(\tilde{\Omega})} \leq C_{*} H^{-1}\|\psi\|_{L^{2}(\Omega)},
$$

where $C_{*}$ depends on $\tilde{\Omega}$.

We mention some additional properties that follow along the proof of Theorem 1.3 ( see e.g. [4, Thm $1.2 \&$ p. 6636]). Firstly, we have the improved estimate for the magnetic energy

$$
\|\operatorname{curl}(\mathcal{A}-\mathbf{F})\|_{L^{2}(\tilde{\Omega})}=\mathcal{O}\left(\kappa^{-1 / 8}\right),
$$


and also for the energy of $\psi$,

$$
\mathcal{G}_{0}(\psi, \mathcal{A}):=\int_{\Omega}\left(|(\nabla-i \kappa H \mathcal{A}) \psi|^{2}-\kappa^{2}|\psi|^{2}+\frac{\kappa^{2}}{2}|\psi|^{4}\right) d x=\mathrm{E}^{\text {asy }}(b, \ell) \kappa^{2}+o\left(\kappa^{2}\right) .
$$

We infer from (1.18) that $\mathcal{G}_{0}(\psi, \mathcal{A})=-\frac{\kappa^{2}}{2}\|\psi\|_{L^{4}(\Omega)}^{4}$, which eventually yields the following formula for the $L^{4}$-energy of the order parameter,

$$
\|\psi\|_{L^{4}(\Omega)}^{4} \leq-2 \mathrm{E}^{\text {asy }}(b, \ell)+\mathcal{O}\left(\kappa^{-1 / 8}\right) .
$$

6.5. Application: The Dirichlet Laplacian. Assuming the hypothesis in Theorem [1.2 on the domain $\Omega$, we will derive an asymptotic upper bound on the eigenvalue $\lambda(\sigma, \mathbf{A} ; \Omega)$, by constructing a trial state related to the GL order parameter.

Under the hypothesis in Theorem 1.2, it is sufficient to handle the case where the domain $\Omega$ consists of a single connected component. In fact, by the min-max principle, $\lambda(\sigma, \mathbf{A} ; \Omega)=$ $\min _{1 \leq i \leq N} \lambda\left(\sigma, \mathbf{A} ; \Omega_{i}\right)$

In the sequel, we assume that $\Omega$ is connected and its boundary consists of a finite number of connected components (as in Sec. 6). Recall the divergence free magnetic potential, F, introduced in (1.13). In light of the relation (1.14), we observe that

$$
\lambda(\sigma, \mathbf{A} ; \Omega)=\lambda(\sigma, \mathbf{F}):=\inf _{u \in H^{1}(\Omega) \backslash\{0\}} \frac{\|(\nabla-i \sigma \mathbf{F}) u\|_{L^{2}(\Omega)}^{2}}{\|u\|_{L^{2}(\Omega)}^{2}} .
$$

The hypothesis $m_{0}(B ; \Omega)>0$ yields that $B(x) \geq c>0$ a.e. on $\Omega$, where $c=m_{0}(B ; \Omega)>0$ is constant. This allows us to benefit from the results and the analysis of Sec. 6 .

6.5.1. Link with the GL energy. In the sequel, we set

$$
\ell=\ell_{\sigma}:=\sigma^{-3 / 8} \text {. }
$$

We fix $a \in(0,1)$ and introduce the parameters

$$
b=\frac{1-a}{m_{0}(B ; \Omega)}, \quad \kappa=b^{-1 / 2} \sigma^{1 / 2} \quad \text { and } \quad H=b \kappa .
$$

The conditions in (6.18) and (6.19) ensure that, as $\sigma \rightarrow+\infty$, the configuration $(\kappa, H, \ell, b)$ satisfies the requirements for using Theorem 1.3 . In particular,

$$
\mathrm{E}(\kappa, H)=\kappa^{2} \mathrm{E}^{\text {asy }}(b, \ell)+\mathcal{O}\left(\kappa^{15 / 8}\right) .
$$

Furthermore, by Remark 1.4 and (1.28), there exist constants $c_{a}^{\prime}>c_{a}>0$ such that

$$
-c_{a} \leq \mathrm{E}^{\mathrm{asy}}(b, \ell) \leq-c_{a}^{\prime}
$$

and

$$
c_{a}, c_{a}^{\prime}=\mathcal{O}(a) \quad\left(a \rightarrow 0_{+}\right) .
$$

Next we pick a minimizing configuration $(\psi, \mathcal{A})_{\kappa, H}$. Collecting (6.21) and (6.16), we obtain

$$
2 c_{a} \leq\|\psi\|_{L^{4}(\Omega)}^{4} \leq 2 c_{a}^{\prime} .
$$

Consequently, since $|\psi| \leq 1$ everywhere, we get

$$
\int_{\Omega}|\psi|^{2} d x \geq \int_{\Omega}|\psi|^{4} d x \geq 2 c_{a}>0 .
$$


Remark 6.3. Fix an arbitrary positive number $\varepsilon<1$. Let us assume that $\mathrm{E}^{\text {asy }}(b, \ell) \underset{\ell \rightarrow 0_{+}}{=} o(1)$ and set $n_{\ell}=\operatorname{Card} \mathcal{J}_{\ell}^{\prime}$ where $\mathcal{J}_{\ell}^{\prime}:=\left\{x \in \mathcal{J}_{\ell}, b B_{\mathrm{av}}^{\ell}(x) \leq 1-\varepsilon\right\}$ and $\mathcal{J}_{\ell}$ is introduced in (1.22). Since $g \leq 0$ and monotone increasing,

$$
o(1)=\mathrm{E}^{\mathrm{asy}}(b, \ell):=\ell^{2} \sum_{x \in \mathcal{J}_{\ell}} g\left(b B_{\mathrm{av}}^{\ell}(x)\right) \leq \ell^{2} \sum_{x \in \mathcal{J}_{\ell}^{\prime}} g\left(b B_{\mathrm{av}}^{\ell}(x)\right) \leq \ell^{2} n_{\ell} g(1-\varepsilon) \leq 0,
$$

hence, $n_{\ell}=o\left(\ell^{-2}\right)$. This yields, by Lebesgue's differentiation theorem, that $b B(z) \geq 1-\varepsilon$ a.e. in $\Omega$. In fact, if we pick $z \in \Omega$ and $\delta_{1}(z)>0$ so that $D\left(z, \delta_{1}(z)\right) \subset \Omega$, then for any fixed $\delta \in\left(0, \delta_{1}(z)\right)$, we have $\int_{D(z, \delta)} b B(y) d y \geq(1-\varepsilon)|D(z, \delta)|$, since

$$
\int_{D(z, \delta)} b B(y) d y \geq \sum_{\substack{x \in \mathcal{J}_{\ell} \backslash \mathcal{J}_{\ell}^{\prime} \\ Q_{\ell}(x) \subset D(z, \delta)}} \int_{Q_{\ell}(x)} b B(y) d y \geq(1-\varepsilon) \ell^{2} \mathcal{N}_{\ell}
$$

where $\mathcal{N}_{\ell}=\operatorname{Card}\left(\left\{x \in \mathcal{J}_{\ell} \backslash \mathcal{J}_{\ell}^{\prime}, Q_{\ell}(x) \subset D(z, \delta)\right\}\right)=|D(z, \delta)| \ell^{-2}+o\left(\ell^{-2}\right)$ as $\ell \rightarrow 0$.

6.5.2. The trial state. We introduce a cut-off function $\chi_{\ell} \in C_{c}^{\infty}(\Omega)$ in order to produce a trial state in $H_{0}^{1}(\Omega)$. We choose $\chi_{\ell}$ such that

$$
\chi_{\ell}(x)=1 \text { for } \operatorname{dist}(x, \partial \Omega)>2 \ell, \quad \text { and } 0 \leq \chi_{\ell} \leq 1,\left|\nabla \chi_{\ell}\right| \leq C_{0} \ell^{-1} \text { in } \Omega .
$$

Using (1.18), we check that

$$
\begin{aligned}
\left\|(\nabla-i \kappa H \mathcal{A})\left(\chi_{\ell} \psi\right)\right\|_{L^{2}(\Omega)}^{2} & =\operatorname{Re}\left\langle-(\nabla-i \kappa H \mathcal{A}) \psi, \chi_{\ell}^{2} \psi\right\rangle_{L^{2}(\Omega)}+\left\|\psi \nabla \chi_{\ell}\right\|_{L^{2}(\Omega)}^{2} \\
& \leq \kappa^{2}\left\|\chi_{\ell} \psi\right\|_{L^{2}(\Omega)}^{2}+C_{0}^{2} \ell^{-2}\|\psi\|_{L^{2}(\Omega)}^{2} .
\end{aligned}
$$

By the simple identity $\mathcal{A}=\mathbf{F}+(\mathcal{A}-\mathbf{F})$ and Cauchy's inequality, we write, for any $\delta \in(0,1)$,

$$
\left\|(\nabla-i \kappa H \mathcal{A})\left(\chi_{\ell} \psi\right)\right\|_{L^{2}(\Omega)}^{2} \geq(1-\delta)\left\|(\nabla-i \kappa H \mathbf{F})\left(\chi_{\ell} \psi\right)\right\|_{L^{2}(\Omega)}^{2}-\delta^{-1} \kappa^{2} H^{2}\|(\mathcal{A}-\mathbf{F}) \psi\|_{L^{2}(\Omega)}^{2} .
$$

We estimate the term $\|(\mathcal{A}-\mathbf{F}) \psi\|_{L^{2}(\Omega)}^{2}$ by using Hölder's inequality, and the two estimates in (6.15) and (6.23); eventually, we get

$$
\|(\mathcal{A}-\mathbf{F}) \psi\|_{L^{2}(\Omega)}^{2} \leq\|\mathcal{A}-\mathbf{F}\|_{L^{4}(\Omega)}^{2}\|\psi\|_{L^{4}(\Omega)}^{2} \leq \frac{C}{H^{2}}\left(2 c_{a}^{\prime}\right)^{1 / 2} \int_{\Omega}|\psi|^{2} d x
$$

We insert this into (6.26) to get (note that $(1-\delta)^{-1} \leq 2$ )

$$
\left\|(\nabla-i \kappa H \mathcal{A})\left(\chi_{\ell} \psi\right)\right\|_{L^{2}(\Omega)}^{2} \geq(1-\delta)\left(\left\|(\nabla-i \kappa H \mathbf{F})\left(\chi_{\ell} \psi\right)\right\|_{L^{2}(\Omega)}^{2}-2 \kappa^{2} \delta^{-1} C\left(2 c_{a}^{\prime}\right)^{1 / 2} \int_{\Omega}|\psi|^{2} d x\right) .
$$

Now we infer from (6.25),

$$
\left\|(\nabla-i \kappa H \mathbf{F})\left(\chi_{\ell} \psi\right)\right\|_{L^{2}(\Omega)}^{2} \leq \frac{1}{1-\delta}\left(\kappa^{2}\left\|\chi_{\ell} \psi\right\|_{L^{2}(\Omega)}^{2}+C_{0}^{2} \ell^{-2}\|\psi\|_{L^{2}(\Omega)}^{2}\right)+2 \kappa^{2} \delta^{-1} C\left(2 c_{a}^{\prime}\right)^{1 / 2} \int_{\Omega}|\psi|^{2} d x .
$$

By (6.19), $\kappa H=\sigma$. Then, in light of (6.17), we deduce that

$$
\lambda(\sigma, \mathbf{F}) \leq \frac{\kappa^{2}}{1-\delta}+\left(\frac{C_{0}^{2}}{1-\delta} \ell^{-2}+2 \kappa^{2} \delta^{-1} C\left(2 c_{a}^{\prime}\right)^{1 / 2}\right) \frac{\|\psi\|_{L^{2}(\Omega)}^{2}}{\left\|\chi_{\ell} \psi\right\|_{L^{2}(\Omega)}^{2}} .
$$

Since $\chi_{\ell}=1$ on $\{\operatorname{dist}(x, \partial \Omega)>2 \ell\}$, we get from (6.24) a constant $M_{a}>0$ such that,

$$
\left\|\chi_{\ell} \psi\right\|_{L^{2}(\Omega)}^{2} \geq\left(1-M_{a} \ell\right)\|\psi\|_{L^{2}(\Omega)}^{2} \geq \frac{1}{2}\|\psi\|_{L^{2}(\Omega)}^{2} \text { for } \ell \text { close to } 0 \text {. }
$$

Furthermore, by (6.19), $\kappa^{2}=(1-a)^{-1} m_{0}(B ; \Omega) \sigma$. And by (6.18),$\ell=\sigma^{-3 / 8}$. Therefore, we deduce from (6.27),

$$
\lambda(\sigma, \mathbf{F}) \leq \frac{m_{0}(B ; \Omega)}{(1-\delta)(1-a)} \sigma+\frac{2 C_{0}^{2}}{1-\delta} \sigma^{3 / 4}+\frac{4 C \delta^{-1}\left(2 c_{a}^{\prime}\right)^{1 / 2} m_{0}(B ; \Omega)}{1-a} \sigma .
$$


Taking the successive limits, $\sigma \rightarrow+\infty, a \rightarrow 0_{+}$and $\delta \rightarrow 0_{+}$, we get

$$
\limsup _{\sigma \rightarrow+\infty}\left(\sigma^{-1} \lambda(\sigma, \mathbf{F})\right) \leq m_{0}(B ; \Omega) \text {. }
$$

Note that we make use of (6.22) which ensures that $c_{a}$ vanishes as $a$ approaches 0 .

\section{ACKNOWLEDGMENTS}

The authors would like to thank B. Helffer and N. Raymond for the valuable comments on a preliminary version of this paper, and the anonymous referees for pointing an error in the formulation of Prop. 3.1 and a suggestion to simplify the proof of Thm. 1.2. A.K. is supported by the Lebanese University in the framework of the project "Analytical and Numerical Aspects of the Ginzburg-Landau Model". Part of this work has been carried out at CAMS (Center for Advanced Mathematical Sciences, Beirut). The authors acknowledge its hospitality.

\section{REFERENCES}

[1] A. Aftalion, S. Serfaty. Lowest Landau level approach in superconductivity for the Abrikosov lattice close to HC2. Sel. Math. 13 (2007), pp. 183-202.

[2] K. Attar. The ground state energy of the two dimensional Ginzburg-Landau functional with variable magnetic field. Ann. Henri Poincaré 32, no. 2, (2015), pp. 325-345.

[3] W. Assaad. The breakdown of superconductivity in the presence of magnetic steps. Commun. Contemp. Math. 23, no. 2, (2021) Article ID 2050005, 53 p.

[4] W. Assaad, A. Kachmar. The Influence of magnetic steps on bulk superconductivity. Discrete and Continuous Dynamical Systems. 36, no. 12, (2016), pp. 6623-6643.

[5] W. Assaad, A. Kachmar, M.P. Sundqvist. The distribution of superconductivity near a magnetic barrier. Commun. Math. Phys. 366, (2019), pp. 269-332.

[6] S. Fournais, B. Helffer. Spectral Methods in Surface Superconductivity, vol. 77. Springer, Berlin (2010).

[7] S. Fournais, A. Kachmar. The ground state energy of the three dimensional Ginzburg-Landau functional. Part I : Bulk regime. Commun. Part. Diff. Equations. 38 (2013), pp. 339-383.

[8] S. Fournais, A. Kachmar, X.B. Pan. Existence of surface smectic states of liquid crystals. J. Funct. Anal. 274, no. 3, (2018), pp. 900-958.

[9] B. Helffer, A. Kachmar. The Ginzburg-Landau functional with vanishing magnetic field. Arch. Ration. Mech. Anal. 218, no. 55 (2015).

[10] B. Helffer, A. Kachmar. Decay of superconductivity away from the magnetic zero set. Calc. Var. Partial Differ. Equ. 56, no. 5, (2017), 130 pp.

[11] B. Helffer, A. Morame. Magnetic Bottles in Connection with Superconductivity. J. Funct. Anal. 185, no. 2, (2001), pp. 604-680.

[12] P.D. Hislop, N. Popoff, N. Raymond, M.P. Sundqvist. Band functions in the presence of magnetic steps. Math. Models Methods Appl. Sci. 26, no. 1, (2016), pp. 161-184.

[13] A. Iwatsuka. Magnetic Schrödinger operators with compact resolvent. J. Math. Kyoto Univ. 26, no. 3, (1986) pp. 357-374.

[14] H. Leinfelder, C.G. Simader. Schrödinger operators with singular magnetic vector potentials. Math. Z. 176, (1981), pp. 1-19.

[15] J.P. Miqueu. Eigenstates of the Neumann magnetic Laplacian with vanishing magnetic field. Annales Henri Poincaré. 19, no. 7, (2018), pp. 2021-2068.

[16] X.B. Pan. Analogies between superconductors and liquid crystals: nucleation and critical fields. Advanced Studies in Pure Mathematics (ASPM) 47-2 (2007). Asymptotic Analysis and Singularities. pp. 479-518.

[17] X.B. Pan, K.H. Kwek. Schrödinger operators with non-degenerately vanishing magnetic fields in bounded domains. Trans. Am. Math. Soc. 354, no. 10, (2002), pp. 4201-4227.

[18] N. Raymond. Bound states of the magnetic Schrödinger operator. EMS Tracts in Mathematics, Vol. 27. European Mathematical Society (EMS), Zürich, (2017).

[19] N. Raymond. Sharp Asymptotics for the Neumann Laplacian with variable magnetic field: Case of dimension 2. Annales Henri Poincaré. 10, no. 10, (2009), pp. 95-122.

[20] E. Sandier, S. Serfaty. The decrease of bulk superconductivity close to the second critical field in the Ginzburg-Landau model. SIAM. J. Math. Anal. 34 (2003), pp. 939-956.

Department of Mathematics, Lebanese University, Nabatieh, Lebanon.

Email address: akachmar@ul.edu.1b

Email address: wehbewehbe@gmail.com 